\title{
Benzene formation in the inner regions of protostellar disks
}

\author{
Paul M. Woods and Karen Willacy \\ Jet Propulsion Laboratory, California Institute of Technology, \\ MS 169-506, 4800 Oak Grove Drive, Pasadena, California 91109, USA. \\ Paul.M.Woods@jpl.nasa.gov
}

\begin{abstract}
Benzene $\left(c-\mathrm{C}_{6} \mathrm{H}_{6}\right)$ formation in the inner $3 \mathrm{AU}$ of a protostellar disk can be efficient, resulting in high abundances of benzene in the midplane region. The formation mechanism is different to that found in interstellar clouds and in protoplanetary nebulae, and proceeds mainly through the reaction between allene $\left(\mathrm{C}_{3} \mathrm{H}_{4}\right)$ and its ion. This has implications for PAH formation, in that some fraction of PAHs seen in the solar system could be native rather than inherited from the interstellar medium.
\end{abstract}

Subject headings: astrochemistry — planetary systems: protoplanetary disks

\section{Introduction}

Benzene is the smallest aromatic molecule, and is presumed to be a basic building block in the formation of polycyclic aromatic hydrocarbons (PAHs). PAHs have been observed in protostellar disks around both low-mass T Tauri (Geers et al. 2006) and intermediate-mass Herbig Ae/Be (HAeBe) stars (Acke \& van den Ancker 2004). The number of detections in $\mathrm{T}$ Tauri-type disks is low, although this is to be expected since the incident UV radiation field is several orders of magnitude smaller than in HAeBe stars. Models indicate that the PAH emission comes from surface layers (Habart et al. 2004), leading to speculation as to whether PAHs form in these high UV environments or elsewhere.

Both PAHs and benzene are also seen in protoplanetary nebulae (PPNe) - PAHs in the circumstellar tori and in the bipolar outflows (Matsuura et al. 2004), and benzene in the torus around CRL618 (Cernicharo et al. 2001). However, the processes that form benzene depend on the environment, with different pathways dominating in the interstellar medium (ISM), in PPNe, in planetary atmospheres (Lebonnois 2005) and, as we shall show, in protostellar disks. In this Letter we consider the formation of benzene in a protostellar disk, and the implications this has for PAH formation. 


\section{Benzene formation in different environments}

The gas-phase synthesis of benzene proceeds via different reaction pathways in different environments, depending on physical and chemical conditions. One pervading condition for high abundances of benzene is that of high density $\left(\gtrsim 10^{9} \mathrm{~cm}^{-3}\right)$. Several reaction schemes have been suggested for benzene formation:

The interstellar medium A reaction scheme for the gas-phase formation of benzene was proposed by McEwan et al. (1999) following the measurement and calculation of new rates for radiative association reactions. The formation pathway for benzene they suggested is thus:

$$
\begin{aligned}
\mathrm{C}_{4} \mathrm{H}_{2}^{+}+\mathrm{H} & \longrightarrow \mathrm{C}_{4} \mathrm{H}_{3}^{+}+\mathrm{h} \nu \\
\mathrm{C}_{4} \mathrm{H}_{3}^{+}+\mathrm{C}_{2} \mathrm{H}_{2} & \longrightarrow c-\mathrm{C}_{6} \mathrm{H}_{5}^{+}+\mathrm{h} \nu \\
c-\mathrm{C}_{6} \mathrm{H}_{5}^{+}+\mathrm{H}_{2} & \longrightarrow c-\mathrm{C}_{6} \mathrm{H}_{7}^{+}+\mathrm{h} \nu \\
c-\mathrm{C}_{6} \mathrm{H}_{7}^{+}+e^{-} & \longrightarrow c-\mathrm{C}_{6} \mathrm{H}_{6}+\mathrm{H}
\end{aligned}
$$

and variations of the above (for instance, $\mathrm{C}_{2} \mathrm{H}_{3}$ may replace $\mathrm{C}_{2} \mathrm{H}_{2}$ in reaction 2). This produces moderate amounts $\left(\sim 10^{-9}\right.$, with respect to $\left.\mathrm{H}_{2}\right)$ of benzene in a dense $\left(10^{4} \mathrm{~cm}^{-3}\right)$ interstellar cloud model.

Protoplanetary nebulae Woods et al. (2002, 2003) applied the above reaction scheme to the dense torus $\left(n \sim 10^{9} \mathrm{~cm}^{-3}\right)$ around the protoplanetary nebula CRL618. CRL618 is rich in hydrocarbons, and benzene has been detected in infra-red spectra of this object (Cernicharo et al. 2001). In the highly ionising environment around CRL618 $\mathrm{C}_{4} \mathrm{H}_{3}^{+}$is formed more efficiently via reactions of various ions with acetylene:

$$
\begin{aligned}
\mathrm{HCO}^{+}+\mathrm{C}_{2} \mathrm{H}_{2} & \longrightarrow \mathrm{C}_{2} \mathrm{H}_{3}^{+}+\mathrm{CO} \\
\mathrm{C}_{2} \mathrm{H}_{3}^{+}+\mathrm{C}_{2} \mathrm{H}_{2} & \longrightarrow \mathrm{C}_{4} \mathrm{H}_{3}^{+}+\mathrm{H}_{2} \\
\mathrm{C}_{2} \mathrm{H}_{2}^{+}+\mathrm{C}_{2} \mathrm{H}_{2} & \longrightarrow \mathrm{C}_{4} \mathrm{H}_{3}^{+}+\mathrm{H}
\end{aligned}
$$

followed by reactions 24, The models of Woods et al. (2002, 2003) produced a fractional abundance of benzene of $\sim 10^{-6}$, in good agreement with observations.

\section{Model}

The protostellar disk model we use will be described more fully in a forthcoming publication, Woods \& Willacy (2006). We use a density and dust temperature profile based 
on that of D'Alessio et al. (2001) (Fig. 1). This is a flared accretion disk model, with a maximum dust grain size of $0.25 \mu \mathrm{m}$, similar to the size of interstellar dust. The disk has a mass accretion rate of $\dot{M}=10^{-8} \mathrm{M}_{\odot} \mathrm{yr}^{-1}$ and a surface density of $\Sigma=100 \mathrm{~g} \mathrm{~cm}^{-2}$ at $1 \mathrm{AU}$, whilst the central star has the following properties: $M_{\star}=0.7 \mathrm{M}_{\odot}, T_{\star}=4000 \mathrm{~K}$ and $R_{\star}=2 \mathrm{R}_{\odot}$. Using these profiles, we calculate UV photon fluxes throughout the disk with the ray-tracing component of the model of Yorke \& Bodenheimer (1999). This calculates the UV field due to the central star and the interstellar radiation field (ISRF) and also includes radiation scattering effects. At $10 \mathrm{AU}$ we assume that the UV field due to the central star is 50,000 times the ISRF, in accordance with the observations of Bergin et al. (2003). Despite the differing spectral shapes of the ISRF and a typical T Tauri stellar field, with strong emission lines dominating the T Tauri spectrum (Bergin et al. 2003), the formation of benzene in the very dense and well-shielded region we consider is not critically dependent on our choice of stellar UV field.

Given gas densities, dust temperatures and UV photon fluxes at each point on our grid], we are then able to solve the heating and cooling balance of the gas in a similar manner to Kamp \& van Zadelhoff (2001), Kamp \& Dullemond (2004) and Gorti \& Hollenbach (2004). The gas temperature can be underestimated by up to two orders of magnitude if it is assumed to be equal to the dust temperature throughout the disk, although this is less critical for the optically thick midplane region where benzene forms. Figure 1 shows the range of gas temperatures within the inner $3 \mathrm{AU}$ of the disk (the benzene formation region).

We use a subset of the extensive UMIST Rate99 gas-phase chemical network (Le Teuff et al. 2000), augmenting it with gas-grain interactions (freezeout, thermal desorption) and grain surface reactions so that our reaction set comprises approximately 2400 reactions amongst 200 species. We include species with more carbon atoms than benzene to ensure that chainlengthening does not result in a spurious build-up of benzene. We have selected reactions which are valid in the region $10-500 \mathrm{~K}$; this upper limit is exceeded in the upper regions of the disk where temperatures can reach $\sim 10,000 \mathrm{~K}$, although at such high temperatures molecules are destroyed. Hence we concentrate on the portions of the disk closer to the midplane, less than 2 scaleheights.

The model follows the passage of a parcel of gas as it flows inwards from the outer regions of the disk ( $>35 \mathrm{AU})$, and as such it traces a period in the evolutionary history of the disk. At the outer edge of the disk the parcel has the composition of a million-year old molecular cloud. The parcel then accretes onto the central star over a period of approximately 200000

\footnotetext{
${ }^{1}$ We use a $35 \mathrm{AU} \times 16 \mathrm{AU}$ (radial $\times$ vertical) grid with spacings of $0.5 \mathrm{AU} \times 0.02 \mathrm{AU}$ to model the very inner region of the disk.
} 
years. As discussed by Willacy et al. (1998), the choice of initial abundances has relevatively little effect on the chemistry of most species.

\section{Results}

We find that benzene formation in a protostellar disk is efficient within $3 \mathrm{AU}$ of the central star, and fractional abundances of $\sim 10^{-6}$ are achieved inside a radius of $2 \mathrm{AU}$ (Fig. 2). The most efficient formation mechanism for benzene in this region is due to the reaction between $\mathrm{C}_{3} \mathrm{H}_{4}$ and its ion, $\mathrm{C}_{3} \mathrm{H}_{4}^{+}$, for which the rate has been measured to within $\pm 25 \%$ in the laboratory $\left(k=7.48 \times 10^{-10} \mathrm{~cm}^{3} \mathrm{~s}^{-1}\right.$; Anicich et al. 1984)2 2 . This is followed by neutralisation of the resulting $c-\mathrm{C}_{6} \mathrm{H}_{7}^{+}$ion in collisions with dust grains:

$$
\begin{aligned}
\mathrm{C}_{3} \mathrm{H}_{4}+\mathrm{C}_{3} \mathrm{H}_{4}^{+} & \longrightarrow c-\mathrm{C}_{6} \mathrm{H}_{7}^{+}+\mathrm{H} \\
c-\mathrm{C}_{6} \mathrm{H}_{7}^{+}+\text {grain } & \longrightarrow g-\mathrm{C}_{6} \mathrm{H}_{6}+g-\mathrm{H},
\end{aligned}
$$

where $g$ - signifies a species adsorbed onto a grain surface.

In this reaction scheme, the adsorption energy of benzene becomes an important factor in determining the amount of gaseous benzene. Estimates of this vary wildly: comparison of similar species in Table 4 of Hasegawa \& Herbst (1993) leads to a value of $4730 \mathrm{~K}$, which agrees well with the experiment of Arnett et al. (1988) using a graphite surface $(4752 \mathrm{~K})$. If one takes the approach of Garrod \& Herbst (2006), and adds the adsorption energy of acetylene to four times the adsorption energy of carbon and four times the adsorption energy of hydrogen, one arrives at the value of $7587 \mathrm{~K}$. This figure lies in the upper range of an experiment on a graphite surface by Lozovik et al. (1995, 4840-7840 K). To quantify the effects of these different adsorption energies, we ran one model with a low binding energy of $4750 \mathrm{~K}$, and one model with a higher binding energy of $7580 \mathrm{~K}$. Results show a significant decrease in the extent of the gas-phase benzene distribution with the higher binding energy, as to be expected, with little change in the peak fractional abundance.

The inner, midplane regions of our disk model are very dense (reaching $\sim 10^{14} \mathrm{~cm}^{-3}$ ), possibly dense enough for three-body (termolecular) reactions to have an effect on the chemistry. In order to investigate the effect on benzene production in particular, we ran a third model including a representative three-body reaction:

$$
\mathrm{C}_{3} \mathrm{H}_{3}+\mathrm{C}_{3} \mathrm{H}_{3}+\mathrm{M} \longrightarrow \mathrm{C}_{6} \mathrm{H}_{6}+\mathrm{M},
$$

\footnotetext{
${ }^{2}$ The UMIST reaction network does not make a distinction in this case between isotopomers of $\mathrm{C}_{3} \mathrm{H}_{4}$; however, the experimental data for reaction (8) was obtained using allene, rather than methylacetylene $\left(\mathrm{CH}_{3} \mathrm{CCH}\right)$ or cyclopropene $\left(c-\mathrm{C}_{3} \mathrm{H}_{4}\right)$
} 
where two propargyl radicals collide with M, a third molecule. This has been shown to be a very efficient formation mechanism for benzene when densities are high; see the discussion

of Cherchneff et al. (1992). We have adopted a rate coefficient from chemical models of the Saturnian atmosphere:

$$
k_{\text {ter }}=\frac{k_{\infty} k_{0} n_{0}}{k_{\infty} n_{0}+k_{0}} \quad \mathrm{~cm}^{3} \mathrm{~s}^{-1}
$$

(Moses et al. 2000) where $\mathrm{k}_{\infty}$, the high pressure limit to the reaction rate, was taken to be $1.66 \times 10^{-13} \mathrm{~cm}^{6} \mathrm{~s}^{-1}$ and $\mathrm{k}_{0}$, the low pressure limit, $1 \times 10^{-27} \mathrm{~cm}^{3} \mathrm{~s}^{-1}$ (Wong et al. 2000 ). Inclusion of this reaction in the model did not result in a significant change in the abundance of gas-phase benzene, despite the presence of large fractional abundances of $\mathrm{C}_{3} \mathrm{H}_{3}$ in dense regions. Even adopting the faster rates for $\mathrm{k}_{\infty}$ and $\mathrm{k}_{0}$ from Lebonnois (2005) $\left(1.2 \times 10^{-10} \mathrm{~cm}^{3} \mathrm{~s}^{-1}\right.$ and $6.0 \times 10^{-28} \mathrm{e}^{1680 / T} \mathrm{~cm}^{6} \mathrm{~s}^{-1}$, respectively) had little overall effect.

\section{Discussion}

The region in which benzene is abundant in our model (Fig. 2) is also rich in other organic molecules: high densities mean fast collision timescales with other molecules and with dust grains, and also protection from the strong stellar UV field in this region. These prime conditions for the growth of organic molecules may allow molecules to grow beyond the single aromatic ring of benzene into PAHs, either by substitution of hydrogen by acetylene in the ring (Wong et al. 2000; Moses et al. 2000), or by the dimerisation of benzene $\left(A_{1}\right)$ with successive phenyl rings to produce naphthalene $\left(A_{2}\right)$, phenanthrene $\left(A_{3}\right)$, pyrene $\left(A_{4}\right)$ and successively large PAH molecules (Cherchneff 1996). Benzene itself is unlikely to persist through to the formation of planets: calculations show that benzene has a timescale for UV destruction in the diffuse solar system of only hundreds of years (Allain et al. 1996; Ruiterkamp et al. 2005), meaning that the benzene seen on Jupiter, Saturn and Titan is unlikely to be primordial.

In order for PAHs to form, the constituents must be present in a region warm enough for ring closure to occur. PAHs only seem to form efficiently at temperatures in the region of 900-1 $100 \mathrm{~K}$ (Frenklach \& Feigelson 1989) and not greater. Production may be possible, too, at lower temperatures $(700-900 \mathrm{~K})$ given a high pressure (Helling et al. 1996). The region of benzene production is at a temperature of a few hundred degrees, and sits just below a region of sufficiently high temperature for $\mathrm{PAH}$ formation. Invoking vertical mixing here could raise benzene and other organics into the higher temperature region, as well as push newly-formed PAHs into the surface layers of the disk, where they are observed. Mixing timescales at a few AU are fast enough ( $625 \mathrm{yr}$; Ilgner \& Nelson 2006) to dredge material from the midplane to surface layers within the lifetime of a T Tauri disk $\left(\sim 10^{6} \mathrm{yr}\right)$. 
For PAHs to subsist in the solar system, the rate of growth of the PAH must be greater than the rate of photodissociation. However, once a PAH reaches 30-40 carbon atoms in size, it is effectively safe from photodissociation since at this size the infrared radiative rate of the $\mathrm{PAH}$ dominates the photodissociation rate. In the ISM, a benzene molecule is destroyed (by removal of an acetylene molecule) at a rate of $1.5 \times 10^{-10} \mathrm{~s}^{-1}$, giving a lifetime of around 200 years. The growth time (accretion of an acetylene molecule) is given by $\tau_{\mathrm{gr}}=\left(n_{0} X_{\mathrm{C}_{2} \mathrm{H}_{2}} k_{\mathrm{C}_{2} \mathrm{H}_{2}}\right)^{-1} \mathrm{~s}$ (Allain et al. 1996). Inserting typical values for the abundance of acetylene in the inner part of the disk, $X_{\mathrm{C}_{2} \mathrm{H}_{2}}=10^{-7}$, and the reaction rate for accretion of acetylene, $k_{\mathrm{C}_{2} \mathrm{H}_{2}}=10^{-11} \mathrm{~s}^{-1}$, gives a necessary density of $1.5 \times 10^{8} \mathrm{~cm}^{-3}$ for PAH growth from benzene. This tallies with the low abundances of benzene in (low density) interstellar cloud models (see Sect. 2) and the lack of a detection of benzene in the ISM. However, these conditions are met within the inner regions of a protostellar disk, as has been shown in our chemical model (which also includes destruction of benzene by reactions with ions). In fact, the strength of the UV field drops below the interstellar level for much of the midplane region, and will remain at that level for much of the transition towards a debris disk. Assuming a PAH of 40 carbon atoms forms solely through the addition of acetylene to a benzene ring, a PAH could be produced from a benzene molecule on a timescale of approximately fifty years at a density of $10^{10} \mathrm{~cm}^{-3}$. Even though the actual route to PAH formation is likely to be different, this estimate does give a representative timescale and show that PAH production from benzene could possibly be prolific in this type of environment. We will consider the formation of larger aromatics in a future paper.

Having ascertained that benzene can survive for an amount of time long enough for PAHs to form from it, the question arises, do PAHs form in similar regions to which benzene forms? The answer to this is unclear, since the supporting observational evidence is sparse. Of the three detections of PAHs in T Tauri-type disks (Geers et al. 2006), all three disks are thought to have inner dust holes (from SED models). Thus it may just be a selection effect that PAH emission is only detected from the upper layers of disks because of the presence of these dust holes: otherwise the continuum emission swamps any possible PAH emission feature originating from deeper in the disk. Further observations are necessary to confirm the correlation between the presence of benzene and PAHs.

This research was conducted at the Jet Propulsion Laboratory, California Institute of Technology under contract with the National Aeronautics and Space Administration. Support comes from an appointment to the NASA Postdoctoral Program at JPL, administered by ORAU through a contract with NASA. 


\section{REFERENCES}

Acke, B., \& van den Ancker, M. E. 2004, A\&A, 426, 151

Allain, T., Leach, S., \& Sedlmayr, E. 1996, A\&A, 305, 602

Anicich, V. G., Blake, G. A., Jhong, K. K., McEwan, M. J., \& Huntress, W. T. 1984, J. Phys. Chem., 88, 4608

Arnett, E. M., Hutchinson, B. J. \& Healy, M. H. 1988, J. Am. Chem. Soc., 110, 16, 5255

Bergin, E., Calvet, N., D’Alessio, P., \& Herczeg, G. J. 2003, ApJ, 591, L159

Cernicharo, J., Heras, A. M., Tielens, A. G. G. M., Pardo, J. R., Herpin, F., Guélin, M., \& Waters, L. B. F. M. 2001, ApJ, 546, L123

Cherchneff, I. 1996, IAU Symp. 178: Molecules in Astrophysics: Probes \& Processes, 178, 469

Cherchneff, I., Barker, J. R., \& Tielens, A. G. G. M. 1992, ApJ, 401, 269

D’Alessio, P., Calvet, N., \& Hartmann, L. 2001, ApJ, 553, 321

Frenklach, M., \& Feigelson, E. D. 1989, ApJ, 341, 372

Garrod, R. T., \& Herbst, E. 2006, ArXiv Astrophysics e-prints, arXiv:astro-ph/0607560

Geers, V. C., et al. 2006, ArXiv Astrophysics e-prints, arXiv:astro-ph/0609157

Gorti, U., \& Hollenbach, D. 2004, ApJ, 613, 424

Habart, E., Natta, A., \& Krügel, E. 2004, A\&A, 427, 179

Hasegawa, T. I., \& Herbst, E. 1993, MNRAS, 261, 83

Helling, C., Jorgensen, U. G., Plez, B., \& Johnson, H. R. 1996, A\&A, 315, 194

Ilgner, M., \& Nelson, R. P. 2006, A\&A, 445, 223

Kamp, I., \& van Zadelhoff, G.-J. 2001, A\&A, 373, 641

Kamp, I., \& Dullemond, C. P. 2004, ApJ, 615, 991

Le Teuff, Y. H., Millar, T. J., \& Markwick, A. J. 2000, A\&AS, 146, 157

Lebonnois, S. 2005, Planet. Space Sci., 53, 486 
Lozovik, Y. E., Popov, A. M. \& Letokhov, V. S. 1995, J. Phys. Chem., 99, 36, 13480

Matsuura, M., et al. 2004, ApJ, 604, 791

McEwan, M. J., Scott, G. B. I., Adams, N. G., Babcock, L. M., Terzieva, R., \& Herbst, E. 1999, ApJ, 513, 287

Moses, J. I., Bézard, B., Lellouch, E., Gladstone, G. R., Feuchtgruber, H., \& Allen, M. 2000, Icarus, 143,244

Ruiterkamp, R., Peeters, Z., Moore, M. H., Hudson, R. L., \& Ehrenfreund, P. 2005, A\&A, 440,391

Willacy, K., Klahr, H. H., Millar, T. J., \& Henning, T. 1998, A\&A, 338, 995

Wong, A.-S., Lee, A. Y. T., Yung, Y. L., \& Ajello, J. M. 2000, ApJ, 534, L215

Woods, P. M. \& Willacy, K. 2006, ApJ, in preparation

Woods, P. M., Millar, T. J., Herbst, E., \& Zijlstra, A. A. 2003, A\&A, 402, 189

Woods, P. M., Millar, T. J., Zijlstra, A. A., \& Herbst, E. 2002, ApJ, 574, L167

Yorke, H. W., \& Bodenheimer, P. 1999, ApJ, 525, 330 

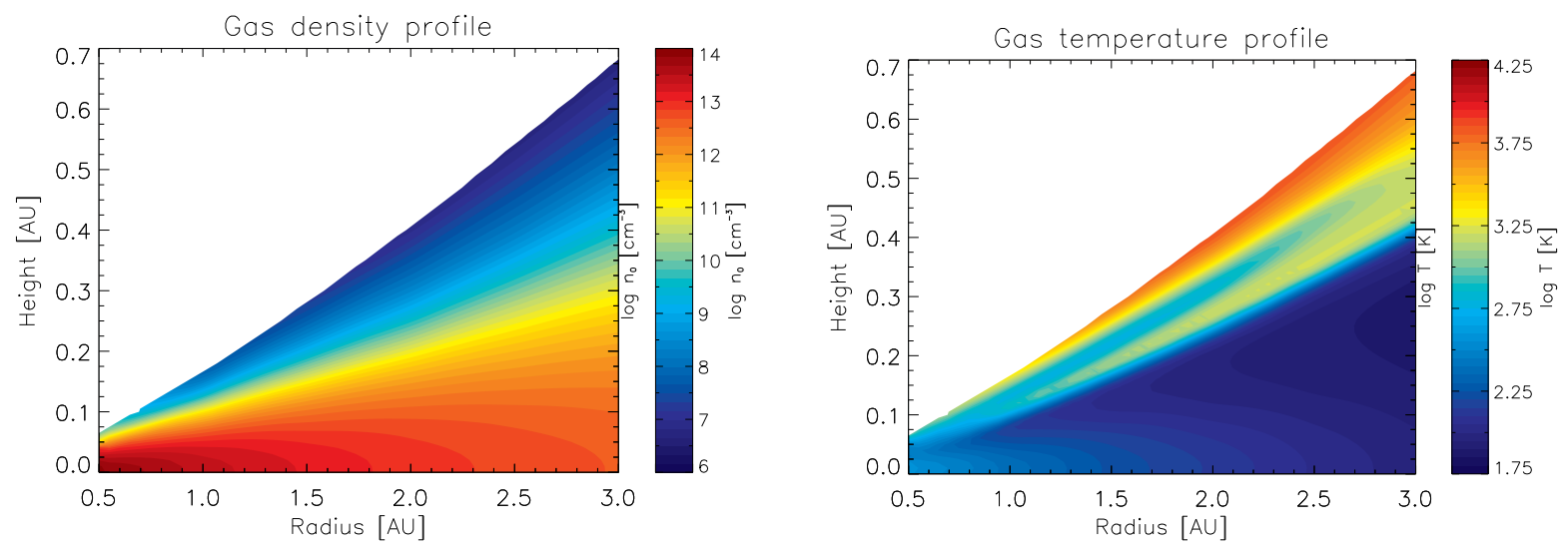

Fig. 1.- Density and gas temperature profiles for the inner $3 \mathrm{AU}$ of the disk model. The left panel shows gas density, whereas the right panel shows the gas temperature calculated by the heating/cooling balance. See the electronic edition of the Journal for a colour version of this figure.
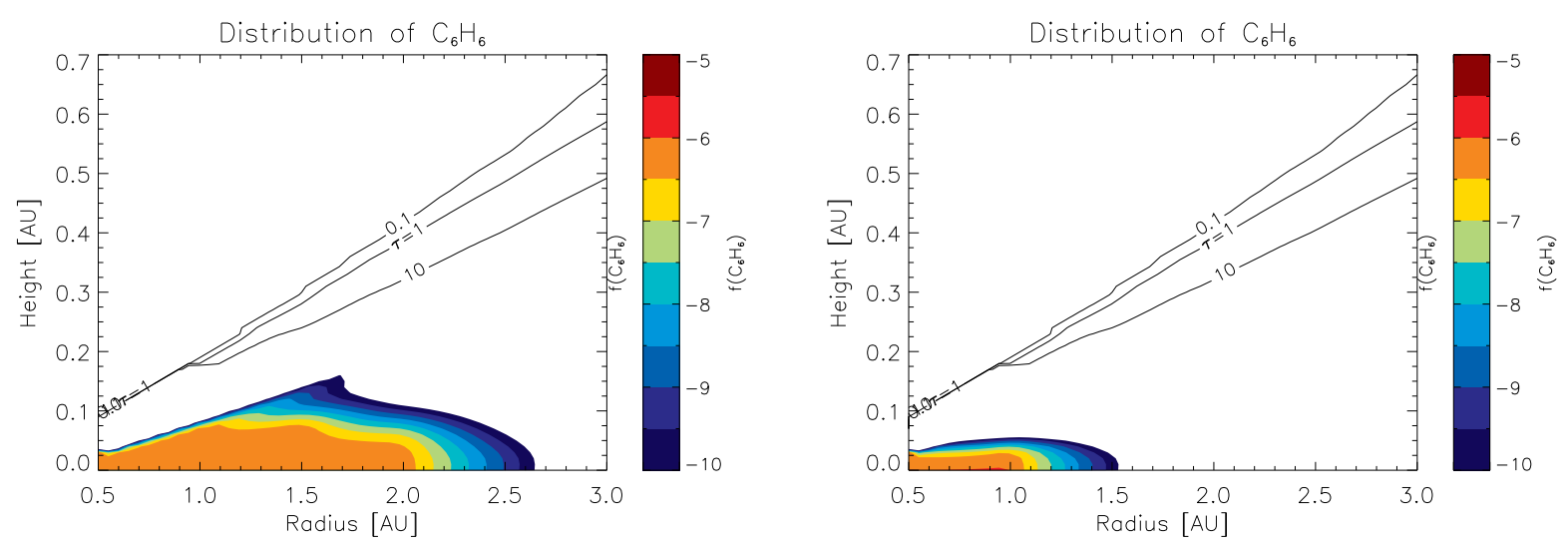

Fig. 2.- The inner $3 \mathrm{AU}$ of the disk, showing the distribution of (gaseous) benzene in terms of fractional abundance. The black solid lines are optical depth $(\tau)$ contours. The left panel shows a model with a low binding energy for benzene, $4750 \mathrm{~K}$. The right panel shows the effect of a higher binding energy, $7580 \mathrm{~K}$. See the electronic edition of the Journal for a colour version of this figure. 